\title{
Physical null conditions: Diameter of a black hole singularity
}

\author{
Leong Ying \\ Thermo Fisher Scientific, Hoboken, USA
}

Email address:

leong.ying@thermofisher.com, klystar@gmail.com

\section{To cite this article:}

Leong Ying. Physical Null Conditions: Diameter of a Black Hole Singularity. American Journal of Modern Physics. Special Issue: New Science Light Path on Cosmological Dark Matters. Vol. 4, No. 1-1, 2015, pp. 42-45. doi: 10.11648/j.ajmp.s.2015040101.18

\begin{abstract}
A black hole is considered a gravitational one-dimensional point of singularity containing an infinite mass within an infinitely small space, and spacetime curves infinitely. Within this singularity all the laws of physics are no longer valid. The physical domains of the large macroscopic scale are described by general relativity and on the small microscopic scale by quantum effects. It is proposed in this new theory that a black hole singularity collapses to a physically defined diameter that perfectly balances between the duality of relativity and quantum states, and in this unified equilibrium condition; physics continues to be obeyed within this non-zero non-infinite black hole singularity.
\end{abstract}

Keywords: Black Hole, Singularity, Null, Zero, Infinite, General Relativity, Quantum Physics, Unified Theory

\section{Introduction}

Einstein's general theory of relativity describes the effects of gravity due to energy-mass curving spacetime. Without going into the detailed derivations or definitions, the famous Einstein field equation can be expressed as:

$$
R_{\mu \nu}-\frac{1}{2} g_{\mu \nu} R+g_{\mu \nu} \Lambda=\frac{8 \pi G}{c^{4}} T_{\mu \nu}
$$

The cosmological constant $\Lambda$ is equivalent to the existence of a non-zero vacuum energy. Penrose-Hawking theorems [1] states that singularity in the solutions of Einstein field equation occurs when matter is compressed into a single infinitely dense space-like point or forms a time-like region of infinite curvature.

Table 1. Schwarzschild radius of objects proportional to their mass

\begin{tabular}{lll}
\hline Objects & Radius $(\mathbf{m})$ & Density $\left(\mathbf{k g} / \mathbf{m}^{\mathbf{3}}\right)$ \\
\hline Earth & 0.009 & $2 \times 10^{30}$ \\
Sun & 3000 & $2 \times 10^{19}$ \\
Milky Way & $2 \times 10^{15}$ & $4 \times 10^{-5}$ \\
Universe & $4 \times 10^{25}$ & $10^{-26}$ \\
\hline
\end{tabular}

The Schwarzschild radius is defined such that when the mass of an object is compressed within a sphere, the escape velocity on the surface equals the speed of light, and any object inside this radius is defined as a black hole. For a non-rotating black hole this radius marks the boundary of the event horizon, from which events trapped within this spacetime cannot affect an outside observer. Table 1 lists the estimated equivalent Schwarzschild radius for various cosmological bodies.

A stellar black hole is formed by the gravitational collapse of a massive star when stellar fusion energy sources are exhausted and can no longer support the inward pressure due to gravity. A white dwarf is a stellar remnant composed mainly of electron-degenerate matter. The Chandrasekhar limit of approximately 1.4 solar masses is the maximum mass of a non-rotating white dwarf, beyond which it cannot be supported by electron degeneracy pressure. The next compressive phase is that of a neutron-degenerate matter star with an upper bound Tolman-Oppenheimer-Volkoff (TOV) limit of approximately 3.0 solar masses. Although theoretically more denser degenerate stars can form, balanced by quark degeneracy pressure, it is assumed that beyond the mass limits of neutron stars the final collapse is to a black hole. It is now commonly interpreted that at the center of galaxies are supermassive black holes, and that such objects are prerequisites to galactic star formations.

Schrödinger equations [2] describe the physical evolution of a quantum state with time, and can be expressed in the general partial derivative form:

$$
i \hbar \frac{\partial}{\partial t} \Psi=\hat{\mathrm{H}} \Psi
$$

Where the Hamiltonian operator $\hat{H}$ characterizes the total 
energy of the given wavefunction $\Psi$ of the quantum state. In classical mechanics the values that characterize a particle such as position and momentum can be determined precisely accordingly to Newton's laws. However, in quantum mechanics, particles do not have precisely determined properties, and when measured, the results form a random probability distribution. Schrödinger equations can predict this probability distribution, but cannot determine the exact result of each measurement. This inherent measurement uncertainty in quantum mechanics is governed by the Heisenberg uncertainty principle that states a particle's position $(x)$ and momentum $(p)$ can only be determined to the following precision of measurements:

$$
\Delta x . \Delta p \geq \frac{\hbar}{2}
$$

The reduced Planck constant or Dirac constant $\hbar=h / 2 \pi$.

Quantum gravity is the theory to describe the force of gravity according to the principles of quantum mechanics. Current understanding of gravity is based on Einstein's general theory of relativity, which is formulated within a classical physics framework and this deterministic approach is incompatible with the uncertainty of a quantum description. Another inconsistency of current theories relate to black hole thermodynamics that forces black holes to contain entropy to obey the second law of thermodynamics when objects pass through the event horizon and all information is lost to an external observer. At the quantum level this manifest itself as Hawking's radiation [3] that theorizes black holes must lose mass due to creations by vacuum fluctuations of virtual particle-antiparticle pairs near to the event horizon. But the most difficult problem to surmount with current theories on black holes is the nature of singularity that cannot accommodate anything, including the laws of physics, into a point space with infinitely curved spacetime.

\section{Duality}

Wave-particle duality states that every elementary particle exhibits the dual properties of a solid-like particle and that of a fluid-like wave. The de Broglie hypothesis links the wavelength $(\lambda)$ of matter to its physical momentum $(p)$ by Planck's constant $(h)$ :

$$
\lambda=\frac{h}{p}
$$

The classic Young's double-slit diffraction experiment demonstrates the dual particle and wave nature of electromagnetic light. For light passing through the two closely spaced openings, an interference diffraction pattern associated with wave properties is observed. However, if the individual photons of light are interacted with and observed, then the detected pattern after passage through the slits collapses into distinct spots associated with particle behaviors.

This dual nature of matter is interpreted by Ying [4] in his universal laws of thermodynamics whereby both energy and entropy are conserved, leading to the birth of parallel twin universes following the big bang moment of creation. Another consequence of zero-balance entropy is to force the anti-symmetric twinned pairs to exist in opposing dual states.

\subsection{Planck Units}

The Planck length $\left(l_{p}\right)$ and mass $\left(m_{p}\right)$ are the smallest dimensions at which classical concepts of gravity and spacetime ceases to be valid and quantum effects dominate. This quantum of length and mass can be defined from three fundamental physical constants:

$$
\begin{gathered}
l_{p}=\sqrt{\frac{\hbar G}{c^{3}}} \\
m_{p}=\sqrt{\frac{\hbar c}{G}}
\end{gathered}
$$

Planck time $\left(t_{p}\right)$ is the period to travel a Planck length at the speed of light. Theoretically these are the smallest discernible values of any observable quantities, and due to the uncertainty principle as defined in equation (3), it is impossible to measure any values smaller than these Planck units. Table 2 lists the various Planck units, with the first five known as the base Planck units and the rests defined as derived Planck units.

Table 2. Planck base and derived units

\begin{tabular}{ll}
\hline Units & SI Values \\
\hline Length $l_{p}$ & $1.616 \times 10^{-35} \mathrm{~m}$ \\
Mass $m_{p}$ & $2.177 \times 10^{-8} \mathrm{~kg}$ \\
Time $t_{p}$ & $5.391 \times 10^{-44} \mathrm{~s}$ \\
Charge $q_{p}$ & $1.876 \times 10^{-18} \mathrm{C}$ \\
Temperature $T_{p}$ & $1.417 \times 10^{32} \mathrm{~K}$ \\
Area $l_{p}{ }^{2}$ & $2.612 \times 10^{-70} \mathrm{~m}^{2}$ \\
Volume $l_{p}{ }^{3}$ & $4.222 \times 10^{-105} \mathrm{~m}^{3}$ \\
Momentum $m_{p} c$ & $6.525 \mathrm{~kg} \cdot \mathrm{m} / \mathrm{s}$ \\
Energy $m_{p} c^{2}$ & $1.956 \times 10^{9} \mathrm{~J}$ \\
Density $m_{p} / l_{p}{ }^{3}$ & $5.155 \times 10^{96} \mathrm{~kg} / \mathrm{m}^{3}$ \\
Energy Density $m_{p} c^{2} / l_{p}{ }^{3}$ & $4.633 \times 10^{113} \mathrm{~J} / \mathrm{m}^{3}$ \\
\hline
\end{tabular}

Planck units are unique natural units originating only from universal parameters associated with fundamental physical theories: speed of light $(c)$ with special relativity and electromagnetism, gravitation constant $(G)$ with general relativity and Newtonian gravity, $\hbar$ with quantum mechanics, electric permittivity $\left(\varepsilon_{0}\right)$ with electrostatics, and Boltzmann constant $\left(k_{B}\right)$ with statistical thermodynamics. In principle, we can define the Planck units as the boundary between the measureable values of particle-like conditions with associated point acting force parameters such as defined in equation (1) and wave-like conditions where there is only a probabilistic existence of a wavefunction as defined in equation (2) with an associated field that permeates all of spacetime. This transition between the deterministic domains and quantum uncertainty is in this author's coming interpretation fundamentally linked to the wave-particle duality existence of black holes.

\subsection{Null Conditions}

In mathematics the null $(\varnothing)$ value is related to an exact 
value of zero or having zero members in a set. In physics a null point is a value in a field where two or more opposing quantities completely cancels each other.

The ground state is the minimum energy of a physical system, but due to the uncertainty principle even this ground state has an associated oscillatory zero-point energy.

In statistical thermodynamics the entropy $(S)$ of a large system that occupies a number of microstates $(\Omega)$ is defined as:

$$
S-S_{0}=k_{B} \ln \Omega
$$

Referenced from absolute zero $\left(S_{0}\right)$ counting states. In a perfect crystal with a unique ground state the entropy approaches zero as the absolute zero temperature is reached. A singularity can be considered the absolute perfect point crystal and hence should have zero entropy associated with it. A similar analogy could be made that a quantized wavefunction that occupies an infinite number of microstates throughout all of spacetime would have an effective infinite entropy. Using this description, the duality flipping between a particle and a wave would suggest entropy switching between zero and infinity. To avoid this non-conserving contradiction, Ying [4] proposed twin universes where the duality flipping is mirrored but in opposing states so that perfectly balanced entropy (and energy) are conserved in a total universal frame of reference. This proposed theory was also able to numerically determine values of negative vacuum energy associated with an accelerating expansion of the universe and the matter-antimatter ratio that are consistent with experimental observations $[5,6]$.

\section{Black Hole Diameter}

\subsection{Spacetime Curvature}

As predicted by Einstein's field equation positive energy-mass causes a localized inward curvature of 4-dimensional spacetime. In a 10-dimensional twin universes existence it is an equivalent possibility for negative energy-mass to curve spacetime outwards.

Table 3. Isolated white star properties in twin universes

\begin{tabular}{ll}
\hline Positive Universe & Negative Universe \\
\hline Visible particle state & Invisible wave state \\
Positive energy $E_{W S}$ & Negative energy $-E_{W S}$ \\
Positive entropy $S_{W S}$ & Negative entropy $-S_{W S}$ \\
Condensed positive energy-mass state & Diffused negative energy-mass \\
curves spacetime inwards & field has no effect on spacetime \\
Dynamic stellar nuclear fusion emits & Dynamic stellar nuclear fusion \\
positive-energy wave that reduces & emits gravitationally repulsive \\
mass of gravitationally attractive & negative-energy particles that \\
condensed star & expand surrounding spacetime \\
White star decreases positive energy & Twinned white star decreases \\
and entropy with time & negative energy and entropy with \\
Surrounding positive universe & time \\
increases positive energy and entropy & Surrounding negative universe \\
with time & increases negative energy and \\
\hline
\end{tabular}

Figure 1 illustrates spacetime curvature in this twin universes perspective noting that the universal conservation of entropy forces the identical twinned matter states to exist in opposing wave-particle identities [4]. Table 3 lists the properties of the isolated condensed white star existing in the positive side of the universe where all quantities and processes have positive values and its parallel duality twin existing in the opposing negative universe.

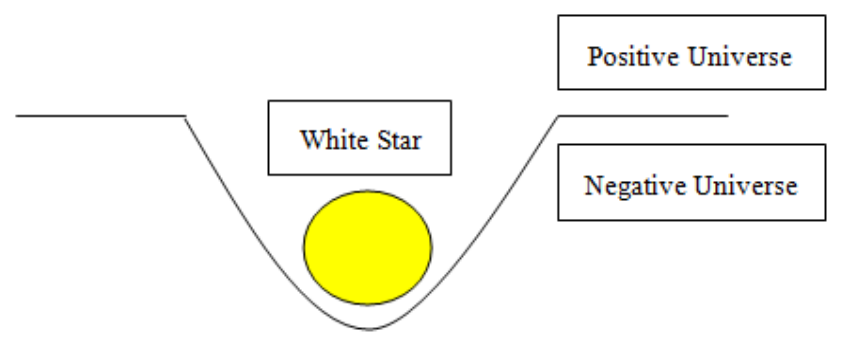

Figure 1. Curvature of spacetime caused by condensed white star

As a massive white star exhausts its supply of fusion elements and can no longer support the gravitational collapse of itself due to the positive energy-mass, it will physically shrink through its various degenerate phases. With sufficient mass the collapse will continue until it passes beyond its own event horizon and turns into a black hole. Figure 2 illustrates the duality flipping of a massive white star on one side of the universe to a black hole on the other side of the negative universe. Table 4 lists the properties of the isolated black hole and its duality twin.

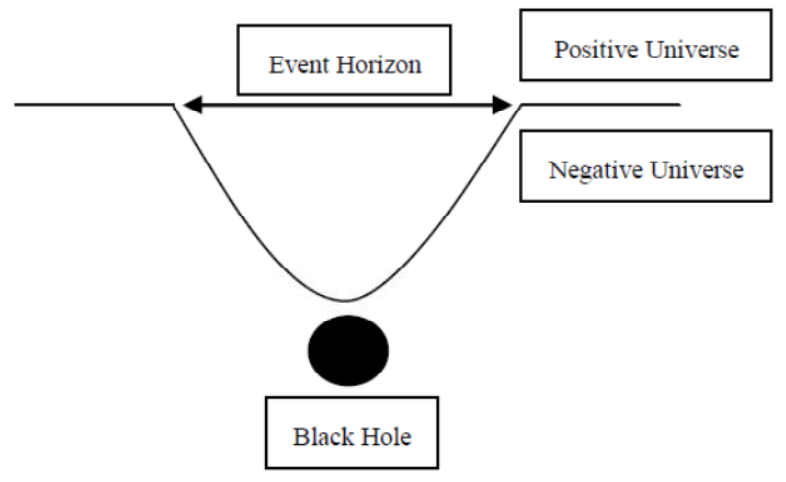

Figure 2. Curvature of spacetime caused by condensed black hole

Table 4. Isolated black hole properties in twin universes

\begin{tabular}{ll}
\hline Positive Universe & Negative Universe \\
\hline Invisible wave state & Visible condensed black hole \\
Positive energy $E_{B H}$ & Negative energy $-E_{B H}$ \\
Positive entropy $S_{B H}$ & Negative entropy $-S_{B H}$ \\
Diffuse positive energy-mass field has & Condensed negative energy-mass \\
no effect on spacetime & state curves spacetime outwards \\
Static non-changing conditions & Static non-changing conditions \\
\hline
\end{tabular}

\subsection{Planck Diameter of Black Hole}

Once a massive white star collapses beyond its own event horizon it transform on the positive observable side of the universe from a physical state obeying the deterministic gravitational laws as defined in equation (1) into the quantum realm whereby it dissipates into a superimposed wavefunction 
that is now governed by quantum probabilistic behaviors as defined by equation (2). To conserve universal thermodynamic properties, the identical twin states on the negative side of the universe simultaneously flips and a black hole with negative energy-mass curves outward the common membrane on which both universes reside. To an observer on the positive universe it does appear that the white star has collapsed into an invisible black hole. However, the most important consequence of this described phenomenon is that at no time is spacetime curved infinitely to produce a point of singularity where all laws of physics breaks down. Both the white star and its progeny black hole maintains physical form through this transformation and thereby will continue to obey all laws of physics, albeit with positive and negative values on either side of the universe, which mathematically and logically is allowed.

In our positive quantified realm the Planck units defines the smallest physical values that are in principle observable, and beyond the Planck limits it collapses into the unobservable quantum realm. This is the condition that defines the physical effective size of a black hole as it flips into existence from the parent white star. Because the black hole appears on the negative side of the universe with negative energy-mass, the repulsive gravity creates its own self-induced pressure to maintain the volumetric integrity of the black hole.

The theoretical density of a 3-dimensional spatial spherical Planck volume where the radius $r_{p}=l_{p} / 2$ can be expressed as:

$$
\rho_{p}=\frac{6}{\pi} \frac{m_{p}}{l_{p}^{3}}
$$

If we therefore assume that the black hole is a perfect spherical crystal structure with homogeneous density composed of uniformly spaced Planck lattice maintained by an outward gravitationally repulsive pressure, then we can scale the Planck density to determine the effective diameter $(D)$ of a black hole of mass $(M)$ :

$$
D=\sqrt[3]{\frac{M}{m_{p}}} l_{p}
$$

Table 5 lists the theoretical effective diameters of condensed matter objects that exist within this transitional wave-particle duality state defined by the Planck units.
Table 5. Theoretical diameter of objects at Planck limits

\begin{tabular}{ll}
\hline Mass & Diameter \\
\hline Planck mass $m_{p}$ & Planck length $l_{p}$ \\
3 Solar mass black hole & $\sim 10^{-22} \mathrm{~m}$ \\
Universe at moment of creation & $\sim 10^{-15} \mathrm{~m}$ \\
\hline
\end{tabular}

\section{Conclusion}

Based on a thermodynamically conserving twin universes perspective it is possible to interpret the collapse of a massive white star into a black hole that is composed of perfect uniformly spaced crystal lattice of Planck dimensions. A negative-energy pressure maintains the physical shape of the black hole and thereby avoiding the problems with the existence of singularities. Physical laws of physics continue to obey the properties and behaviors of both the positive energy-mass white star and the negative energy-mass black hole either in the particle state govern by relativity or the wave state by quantum mechanics.

\section{References}

[1] S. Hawking and R. Penrose, The nature of space and time, Princeton University Press: Princeton, 1996.

[2] E. Schrödinger, An undulatory theory of the mechanics of atoms and molecules, The Physical Review, vol. 28, no. 6, 1926, pp. 1049-1070.

[3] S. W. Hawking, Black hole explosions?, Nature, vol. 248, 1974, pp. 30-31.

[4] L. Ying, Twin universes: universal laws of thermodynamics, American Journal of Modern Physics. Special Issue: New Science Light Path on Cosmological Dark Matters. Vol. 4, No. 3, 2015, pp. 1-4.

[5] L. Ying, Nuclear fusion drives cosmic expansion, Springer Proceedings in Physics: Springer, vol. 137, 2011, pp. 379-381.

[6] L. Ying, Pi is fundamental cosmic frequency in expanding universe, The Open Astronomy Journal, vol. 6, 2013, pp. 7-9. 